\section{Rapid calculation procedures for the maximum-likelihood method of adaptive psychophysics}

\author{
BRIAN R. SHELTON \\ University of Western Ontario \\ London, Ontario N6A 5C2, Canada
}

A number of psychophysical procedures involve the adaptive presentation of stimuli, in which the response of the subject determines the parameters of subsequent trial presentation. In the case of the determination signal threshold, for example, the signal level is made more or less intense, depending on the accuracy of detection. A number of rules can be used to adjust the signal level, but perhaps the most frequently used are PEST (Taylor \& Creelman, 1967), the adaptive staircase (Levitt, 1971), and maximum-likelihood procedures (Hall, 1974, 1981; Pentland, 1980).

A comparison of the three procedures has revealed that all obtain similar threshold estimates, but that certain characteristics of each method may make one more suitable than another in particular applications (Shelton, Picardi, \& Green, 1982). For instance, PEST is recommended in cases in which a constant precision of measurement is required across a set of measurements, and the maximum-likelihood method is preferable in instances in which there is uncertainty about the number of trials that can be completed in the testing situation.

These procedures have all been previously described and have been successfully used in a variety of situations. The three methods require computer control of stimulus parameters, and several calculations must be made between trials to determine the next presentation in the sequence. The eventual outcome of each procedure is to converge on the stimulus configuration that can be detected or discriminated with a fixed level of accuracy.

In the case of maximum-likelihood procedures, the calculations required between trials can be quite extensive. Pentland (1980) describes the required computer code as trivial, but the process can be time-consuming. In many psychophysical applications, the time between trials is preferred by subjects to be relatively short, on the order of a couple of seconds. In some situations, and certainly with some programming strategies, it is difficult to complete the calculations required in the

The author would like to thank David M. Gieen for his support and Fred Smith for his assistance in the initial stages of this project. Thanks are also given to John Booth and Walter Gilmore for their comments and to Wanda "Ace" Marks for typing the manuscript. This research was financed in part by the National Institute of Health and by the Natural Sciences and Engineering Research Council of Canada. maximum-likelihood procedure fast enough to present trials at a rate that the subjects find comfortable. In this report, two subroutines are provided that have been useful in minimizing the time required to proceed to the next trial. The code has been successfully used with two compiler systems, a PDP-15 and a microcomputer based on the Cromemco single-card computer, in both BASIC and FORTRAN. Interpreter versions of the same code, however, are still too slow to be of any practical use.

The maximum-likelihood procedure requires a finite number of possible stimulus values, bracketing the assumed signal threshold. For each of the possible stimulus values, a psychometric function is assumed that describes the probability of a detection response given that the assumed psychometric describes the "true" psychophysical function. On the presentation of a given stimulus, the probability of the observed response given each of the possible stimulus values is calculated and accumulated with the probabilities recorded on previous trials. As Pentland (1980) has shown, the maximum efficiency is obtained if the most likely threshold value is presented on each trial, so that this value, the current estimate of signal threshold, is the stimulus value presented on each trial.

The two subroutines described here perform this procedure quitc quickly given a minor constraint on the set of stimuli used for presentation. Specifically, the stimuli chosen as possible threshold values must be equally spaced along the physical continuum in question. If this restriction is met, then the psychometric assumed for one stimulus value is exactly the same as that assumed for another, except for a translation along the physical continuum of the psychometric. Therefore, only one psychometric need be calculated, and it need be calculated only once. The outcome of the calcula tions can be stored in an array, and the appropriate value can be recovered by the proper indexing of the information.

There are two subroutines involved. PCALC.FOR sets up the assumed psychometric arrays, and TCALC.FOR uses this information in determining, based on a subject's response, the most probable signal threshold. In the subroutine PCALC.FOR, a modified logistic function was assumed as the psychometric, which has the form $\mathrm{P}_{\mathrm{i}}(\mathrm{c} / \mathrm{t})=.5+\left[.50 /\left(1+\mathrm{e}^{\alpha\left(\mathrm{t}-\mathrm{X}_{\mathrm{i}}\right)}\right)\right]$, to describe the probability of a correct response for each member of the stimulus set $X_{i}$, given a threshold value of $t$. The parameter $\alpha$ controls the slope of the assumed psychometric.

In PCALC.FOR, the psychometric is automatically adjusted so that the probabilities range from .55 to .95 across the specified stimulus set. This characteristic can easily be altered by the assignment of the variable "ALPHA" to the desired value. Further, the form of the 
assumed psychometric can be changed to suit the situation in question. The subroutine PCALC.FOR can be used to set up the proper psychometric for adapting either the signal value or the amplitude of a masker. PVAL $(1,1)$ stores the probability values associated with a correct response, and PVAL $(2,1)$ stores the probability values associated with an incorrect response. The array is converted to a logarithmic representation to make subsequent calculations in the subroutine TCALC.FOR additive rather than multiplicative. The parameter IFLAG determines whether the probabilities will be appropriate to adapt a signal level or a masker level.

The subroutine TCALC.FOR, on the other hand, must be executed after each trial and provides an estimate of the most probable threshold value. Care must be taken to zero PMAX and the array PCUM before a new block of trials is initiated. Also, the variable ILEV refers to the index of the array XLEV, and the actual value of the threshold value is defined by XLEV(ILEV) in the main program. The stimulus chosen as the signal level for the first trial is arbitrary, and it must be assigned in some way (see Shelton et al., 1982). The index of the level chosen, ILEV, is passed to the subroutine TCALC.FOR, and the ILEV retumed is the index for the stimulus to be presented on the subsequent trial. This process is repeated on each trial, until the procedure converges on a threshold estimate. Note that if PCALC.FOR is set to adapt the signal (IFLAG=1), XLEV(ILEV) will refer to a signal level, but the same value refers to a masker level if the subroutine is set to adapt a masker (IFLAG $=-1$ ).

The subroutine TCALC.FOR is the element that determines the rate at which trials can be presented. On a 4-MHz system, the program retums from a call in about $140 \mathrm{msec}$, given 100 possible stimuli. With only 50 possible stimuli, the subroutine returns in about $75 \mathrm{msec}$. The response is fast enough to be useful in most psychophysical applications.

The maximum-likelihood procedure has not been used in a large number of measurement situations. Part of the reason for this, undoubtedly, has been difficulty in implementing the procedure in a manner that is fast enough for some psychophysical paradigms. The subroutines described here allow for the rapid calculation of the appropriate values, which will make the procedure easier to use in a variety of situations. As the procedure is more widely applied, the strengths and weaknesses of the method will become more clearly defined.

Hardware Requirements. Both programs are standard FORTRAN subroutines, with no machine-specific code. The memory required is largely determined by the number of possible stimulus values used in an application. The programs reserve $2 \mathrm{~N}-6$ real array locations, where $\mathrm{N}$ is the number of possible signal or masker levels. In general, this is not a serious constraint.

Availability. The programs are small and constitute only about 30 lines of FORTRAN code. Printouts of the source code are available from the author upon request.

\section{REFERENCES}

HaLt, J. L. Maximum-likelihood sequential procedure for estimation of psychometric functions. Journal of the Acoustical Society of America, 1974, 55, 1090-1091.

Hall, J. L. Hybrid adaptive procedure for estimation of psychometric functions. Journal of the Acoustical Society of America, $1981,69,1763-1769$.

LEviTT, H. Transformed up-down methods in psychoacoustics. Journal of the Acoustical Society of America, 1971, 49, 467. 477.

Pentuand, A. Maximum likelihood estimation: The best PEST. Perception \& Psychophysics, 1980, 28, 377-379.

Shelton, B. R., Picardi, M. C., \& Green, D. M. Comparison of three adaptive psychophysical procedures. Journal of the Acoustical Society of America, 1982, 71, 1527-1533.

Taylor, M. M., \& Creelman, C. D. PEST: Efficient estimates on probability functions. Journal of the Acoustical Society of America, 1967, 41, 782-787.

(Accepted for publication November 20, 1982.) 